\title{
JOURNAL.RU
}

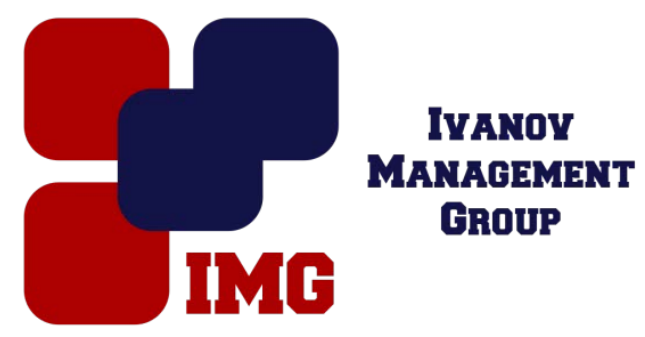

Ле Дык Тиеп, Нгуен Ван Чонг

Московский Авиаиионный Институт

Москва, Россия

doi: 10.18411/lj-28-02-2017-1-07

idsp 000001:lj-28-02-2017-1-07

\section{Улучшенный алгоритм векторной ШИМ для регулирования частоты вращения вала асинхронного двигателя}

\begin{abstract}
Аннотация
В статье предложен алгоритм формирования напряжения на обмотках асинхронного двигателя (АД)в режиме векторной ШИМ прирегулированиичастоты вращения асинхронного двигателя. Предложено использовать в алгоритме формировать дополнительные векторы для алгоритма векторной ШИМ, как способ управления сквозных токов. Приведены результатыимитационногомоделирования предложенного алгоритма векторной ШИМ, которые показали её работоспособность. Приведено способ регулирования частоты вращения асинхронного двигателя, показано что разработанный алгоритм с использованием промежуточных векторов позволил исключить сквозные токи первого рода в инверторе при управления двигателем и уменьшать его колебание скорости, момент и тока статора средством увеличения количества формируемых векторов в каждом секторе.

Ключевые слова: векторная широтно-импульсная модуляция, электропривода синхронного двигателя, трехфазный инвертор, силовой транзисторный ключ.
\end{abstract}

\section{Содержание}

Широтно-импульсная модуляция применяется в преобразовательной технике, как способ формирования импульсного напряжения и регулирования его параметров. Известно большое число различных методов ШИМ, к достоинствам которых следует отнести обеспечение широкого линейного диапазона модуляции; снижение потерь на переключение силовых ключей транзисторов; улучшение спектрального состава и простоту реализации. Один из способов реализации ШИМ при управлении асинхронного двигателя -формирование изображающего вектора напряжения статора. Этот метод применяется в подавляющем большинстве систем с пространственно-векторным формированием ШИМ. 


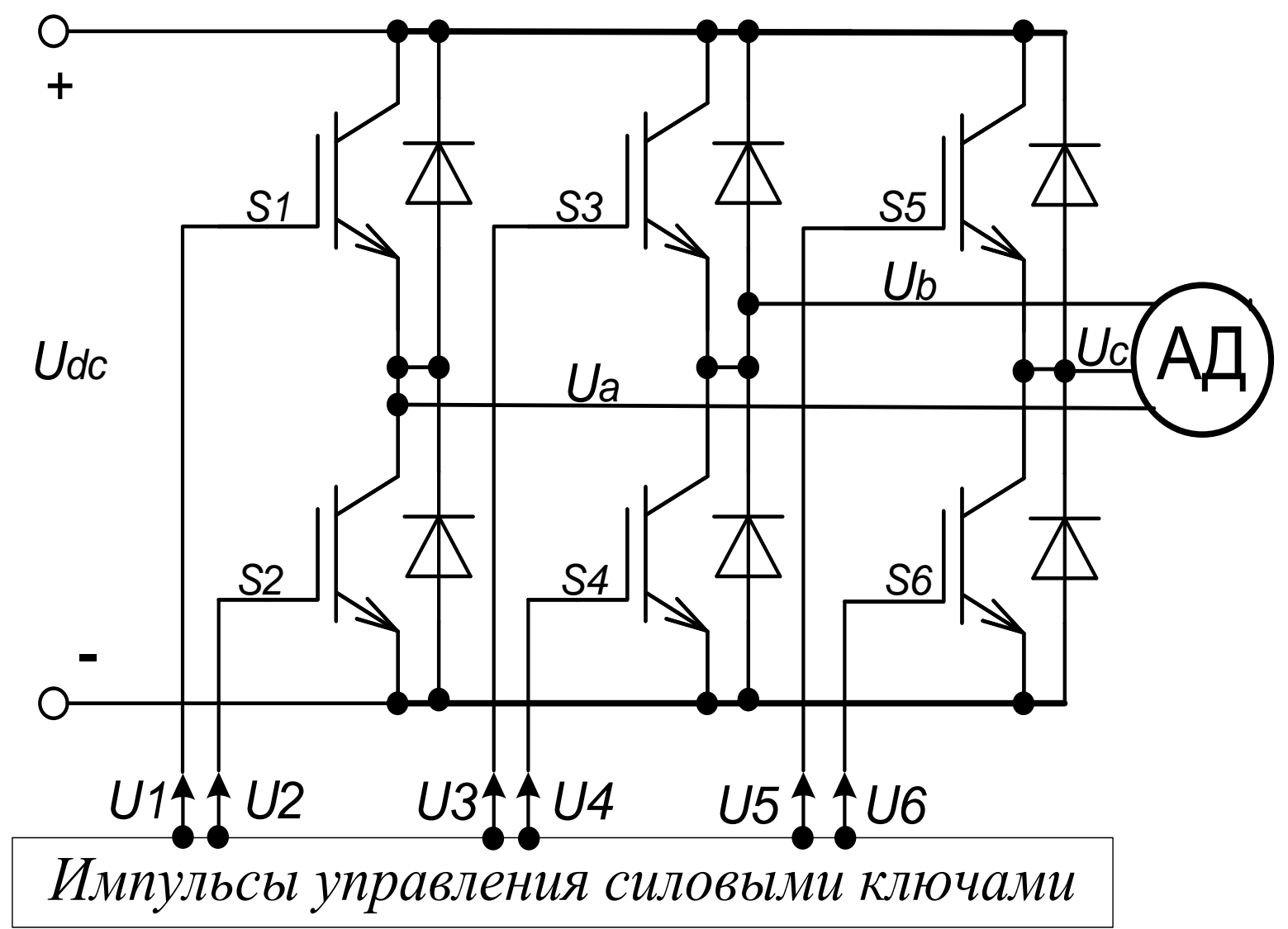

Рис. 1 Структура подключения трехфазного инвертора к АД

Для управления шестью ключами существуют $26=64$ состояния, из которых рабочими является ограниченное число [5, 6, 7, 8].Обозначим их состояния символами Vхуи Vх.

Где:

- $\operatorname{Vx}(\mathrm{x}=0,1 . .7)$ - базовыевекторы;

- Vху- остальные рабочие состояния ключей инвертора; где х-является десятеричным значением двоичных кодов ключей S1S3S5, у -является десятеричным значением двоичных кодов ключей S2S4S6.При традиционном векторном формировании ШИМ $[1,2,3]$ используются 8 состоянии ключей инвертора, которые описываются 8-ю векторами V0, V1, V2, V3, V4, V5, V6, V7. При формировании более одного вектора в секторе (рассмотрим для примера сектор, ограниченный векторами (V4, V6) порядок следования базовых векторов следующий:

$\left(V_{0} \Rightarrow V_{4} \Rightarrow V_{6} \Rightarrow V_{7} \Rightarrow V_{6} \Rightarrow V_{4} \Rightarrow V_{0}\right) \Rightarrow\left(V_{0} \Rightarrow V_{4} \Rightarrow V_{6} \Rightarrow V_{7} \Rightarrow V_{6} \Rightarrow V_{4} \Rightarrow V_{0}\right)$

B секторе (V4, V6) имеют 6 переключений: (V0 $\Rightarrow \mathrm{V} 4)$, (V4 $\Rightarrow \mathrm{V} 6)$, (V6 $\Rightarrow \mathrm{V} 7)$, $(\mathrm{V} 7 \Rightarrow \mathrm{V} 6),(\mathrm{V} 6 \Rightarrow \mathrm{V} 4), \quad(\mathrm{V} 4 \Rightarrow \mathrm{V} 0) . \quad$ В $[9,12]$ было показано, что все эти переключения приводят к возникновению сквозного тока и для исключения сквозного тока в переходах (V4 $\Rightarrow \mathrm{V} 6)$, (V6 $\Rightarrow \mathrm{V} 4)$ предположено использовать дополнительные промежуточные векторы V41, V42, V21, V24, V12, V14. 
Таблица 1

Состояния ключей для формирования дополнительныхопределенных векторов

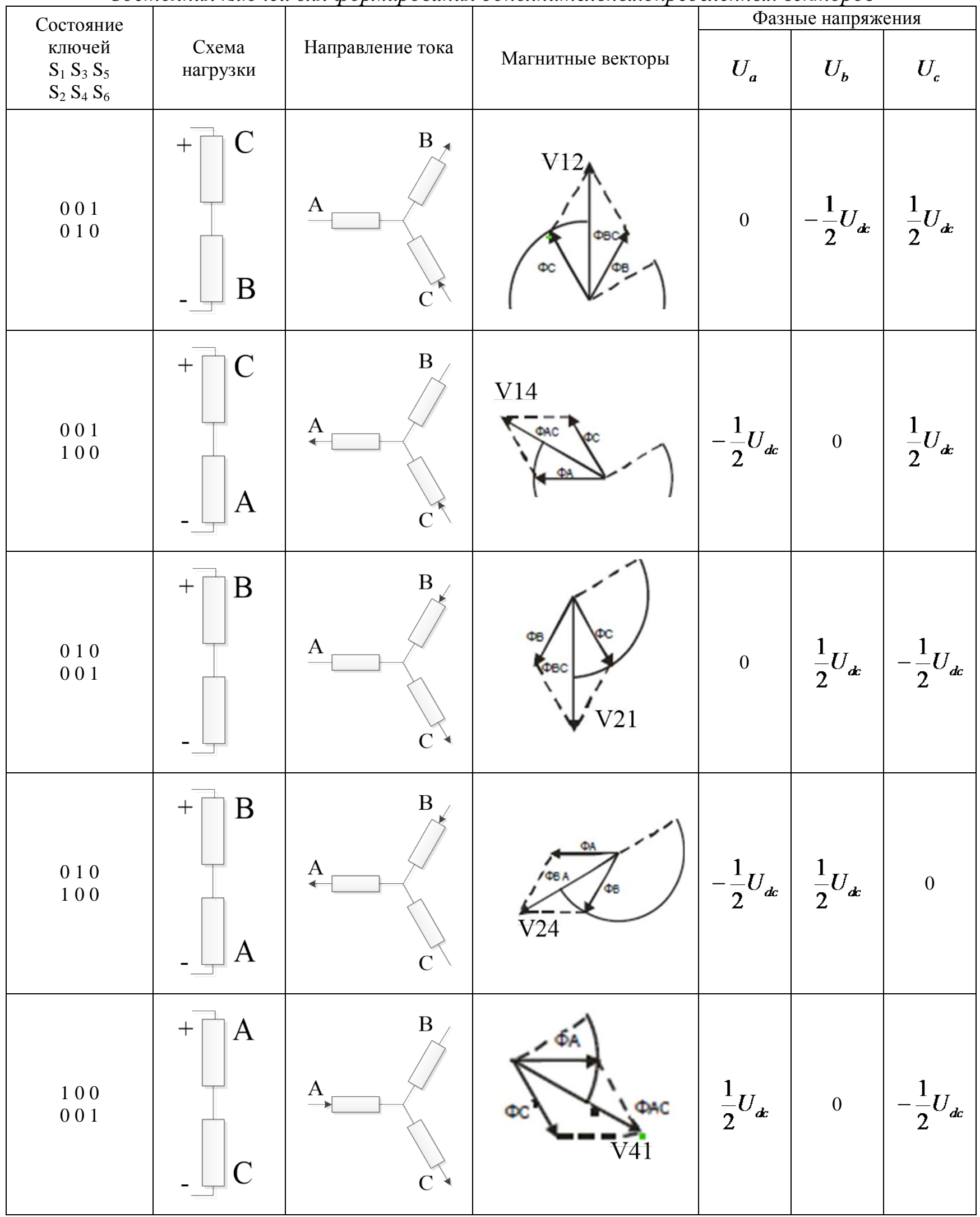



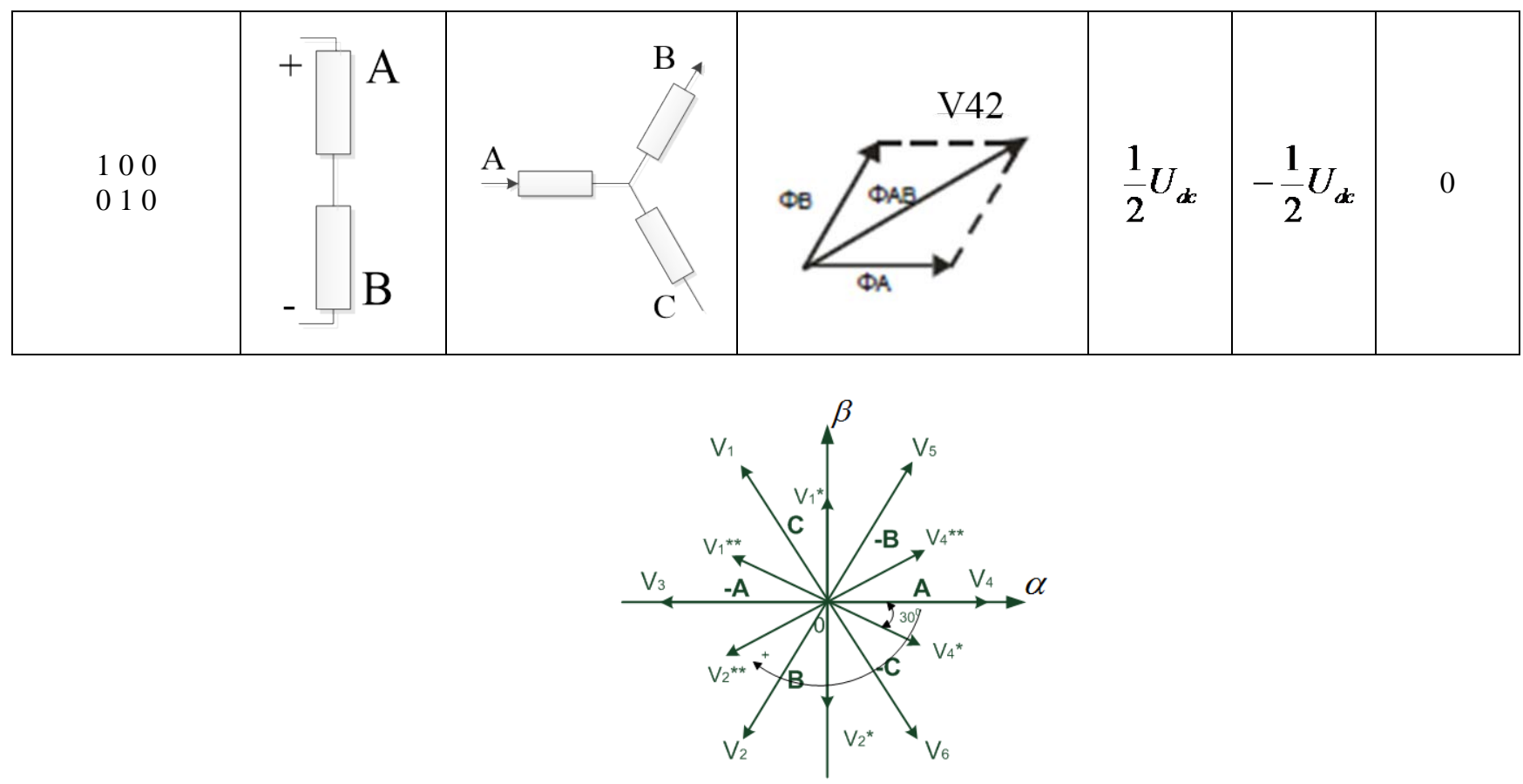

Рис. 2 Результирующце векторы в неподвижной системе координат

Для регулирования частоты вращения вала АД предложен перспективный алгоритм с следующимпорядкомследования базовых векторов при формирования любого вектора напряжения в секторах (V4, V6), (V6, V2), (V2, V3), (V3, V1), (V1, V5), (V5, V4) будет:

$$
\begin{aligned}
& +V_{0} \Rightarrow \mathrm{V}_{4} \Rightarrow V_{41} \Rightarrow \mathrm{V}_{6} \Rightarrow V_{41} \Rightarrow \mathrm{V}_{4} \Rightarrow \mathrm{V}_{0} ; \\
& +V_{0} \Rightarrow \mathrm{V}_{6} \Rightarrow V_{21} \Rightarrow \mathrm{V}_{2} \Rightarrow V_{21} \Rightarrow \mathrm{V}_{6} \Rightarrow \mathrm{V}_{0} ; \\
& +V_{0} \Rightarrow \mathrm{V}_{2} \Rightarrow V_{24} \Rightarrow \mathrm{V}_{3} \Rightarrow V_{24} \Rightarrow \mathrm{V}_{2} \Rightarrow \mathrm{V}_{0} ; \\
& +V_{0} \Rightarrow \mathrm{V}_{3} \Rightarrow V_{14} \Rightarrow \mathrm{V}_{1} \Rightarrow V_{14} \Rightarrow \mathrm{V}_{3} \Rightarrow \mathrm{V}_{0} ; \\
& +V_{0} \Rightarrow \mathrm{V}_{1} \Rightarrow V_{12} \Rightarrow \mathrm{V}_{5} \Rightarrow V_{12} \Rightarrow \mathrm{V}_{1} \Rightarrow \mathrm{V}_{0} ; \\
& +V_{0} \Rightarrow \mathrm{V}_{5} \Rightarrow V_{42} \Rightarrow \mathrm{V}_{4} \Rightarrow V_{42} \Rightarrow \mathrm{V}_{5} \Rightarrow \mathrm{V}_{0} .
\end{aligned}
$$

Так длительность существования промежуточных векторов крайне малпо сравнению сбазовыми векторами, и их влиянием на направление и модуль формируемых векторов можно пренебречь. Соответствующая временная диаграмма переключения силовых ключ при формировании вектора в секторе (V4, V6) дана на рис.3.В остальных секторах формирование будет аналогичным.

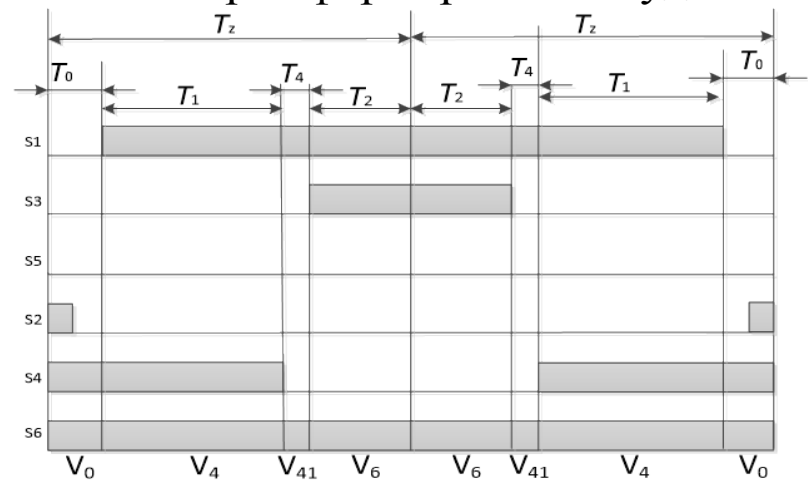

Рис.3 Временная диаграмма переключения силовых ключейпри формировании вектора в секторе (V4, V6) 
Для регулирования частоты вращения вала АД приводим по закону, например $\frac{U}{f}=$ const . Приуменьшения амплитуды колебания тока статора, скорости ротора и момента АД количество формируемых векторов в каждом секторекак правило велико. Число формируемых векторов в секторе будем обозначать $n$.Например формирование трех векторов в секторе (V4, V6) то $n=3$, который приведен на рис. 4. Для исследования влияния параметра $n$ на качество электропривода асинхронного двигателя в режиме векторной ШИМ используем имитационную програмированную модельна Matlab, которая приведена на рис. 5.

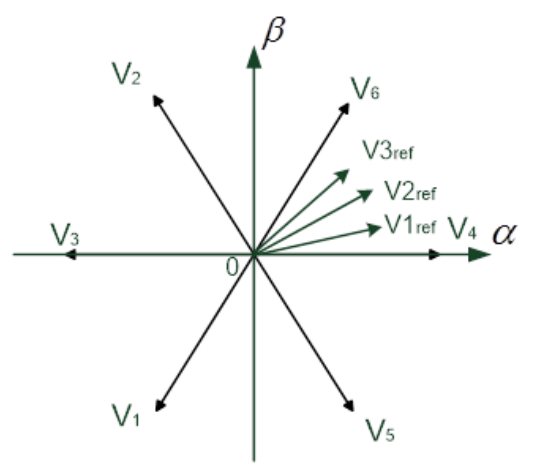

Рис. 4 Формирование трех векторов

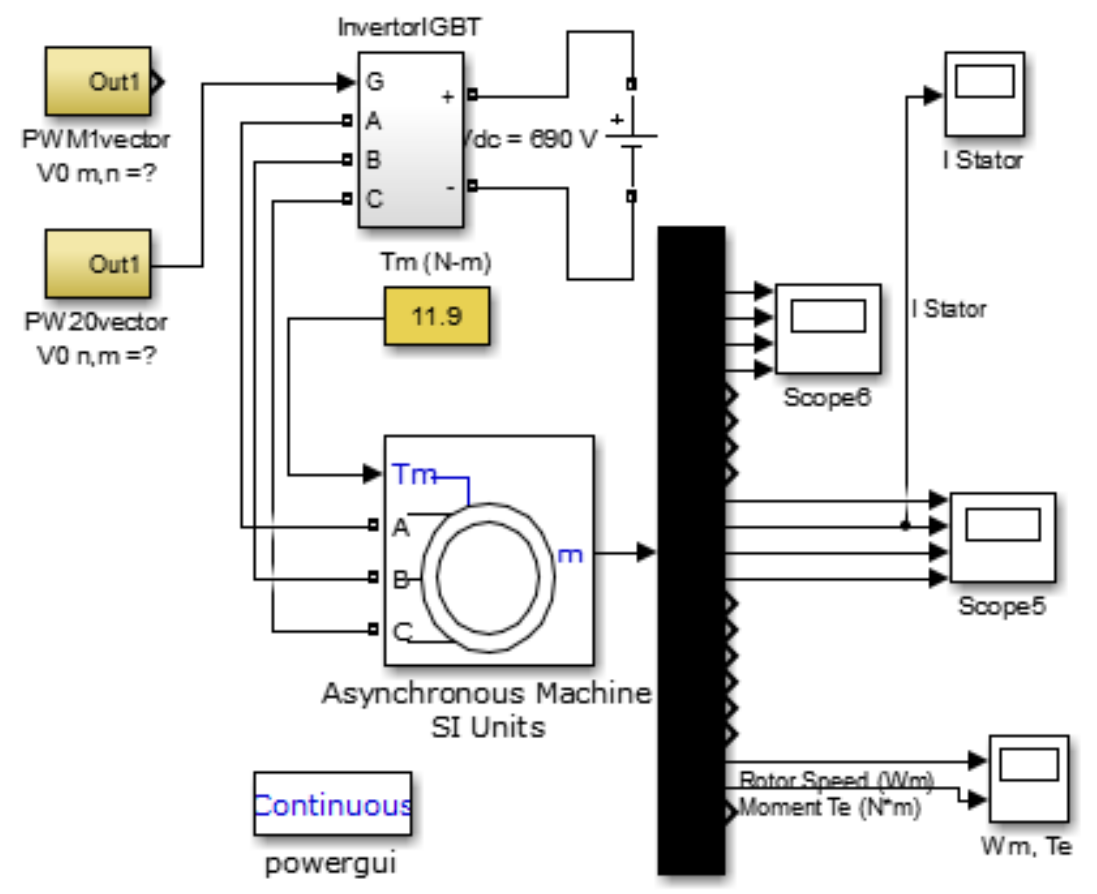

Рис. 5 Схема моделиуправления асинхронным двигателем в режиме векторной ШИМ вMatlabSimulink

Где:

- $\quad$ блок: PWM1vector - Блок программирования формирования одного вектора в каждом секторе;

- блок PW20vector - Блок программирования формирования 20 векторов в каждом секторе; 
Результаты моделирования сведены в табл.2, 3 .

Таблиия 2

Результатов оиенивания качества электропривода при $n=1$ и частоты работы двигателя изменяются 60, 50, 15, 5, 1:

\begin{tabular}{|c|c|c|c|c|c|}
\hline \multicolumn{2}{|c|}{$\mathrm{f}$} & 60 & 15 & 5 & 1 \\
\hline \multirow{4}{*}{$n=1$} & $\omega$, & 187, & 46, & 15, & 2.46, \\
& $\Delta \omega_{(\mathrm{rad})}$ & 0.01 & 15 & 48 & 7.54 \\
\cline { 2 - 6 } & $\Delta M_{(\text {H.M })}$ & 12 & 258 & 259 & 97 \\
\cline { 2 - 7 } & $\Delta I$ (A) & 15.8 & 51.8 & 89.9 & 17 \\
\hline
\end{tabular}

Таблийа 3

Результатов оченивания качества электропривода при $n=20$ и частоты работы двигателя изменяются 60, 50, 15, 5, 1:

\begin{tabular}{|c|c|c|c|c|c|}
\hline \multicolumn{2}{|c|}{$\mathrm{f}$} & 60 & 15 & 5 & 1 \\
\hline \multirow{4}{*}{$n=2$} & $\omega$, & 187, & 46, & 15, & 2.46, \\
& $\Delta \omega^{(\mathrm{rad})}$ & 0 & 0.4 & 1.1 & 1.04 \\
\cline { 2 - 6 } & $\Delta M_{\text {(H.м) }}$ & 6 & 17 & 19 & 28 \\
\cline { 2 - 7 } & $\Delta I$ (А) & 0.625 & 1.84 & 2.6 & 4.5 \\
\hline
\end{tabular}

Где: $\Delta \omega=\omega_{S P W}-\omega_{S i n}, \Delta M=M_{S P W}-M_{S i n}, \Delta I=I_{S P W}-I_{S i n}$

В качестве примера приведены графики при $f=3, n=20$, рис. 6,7 .
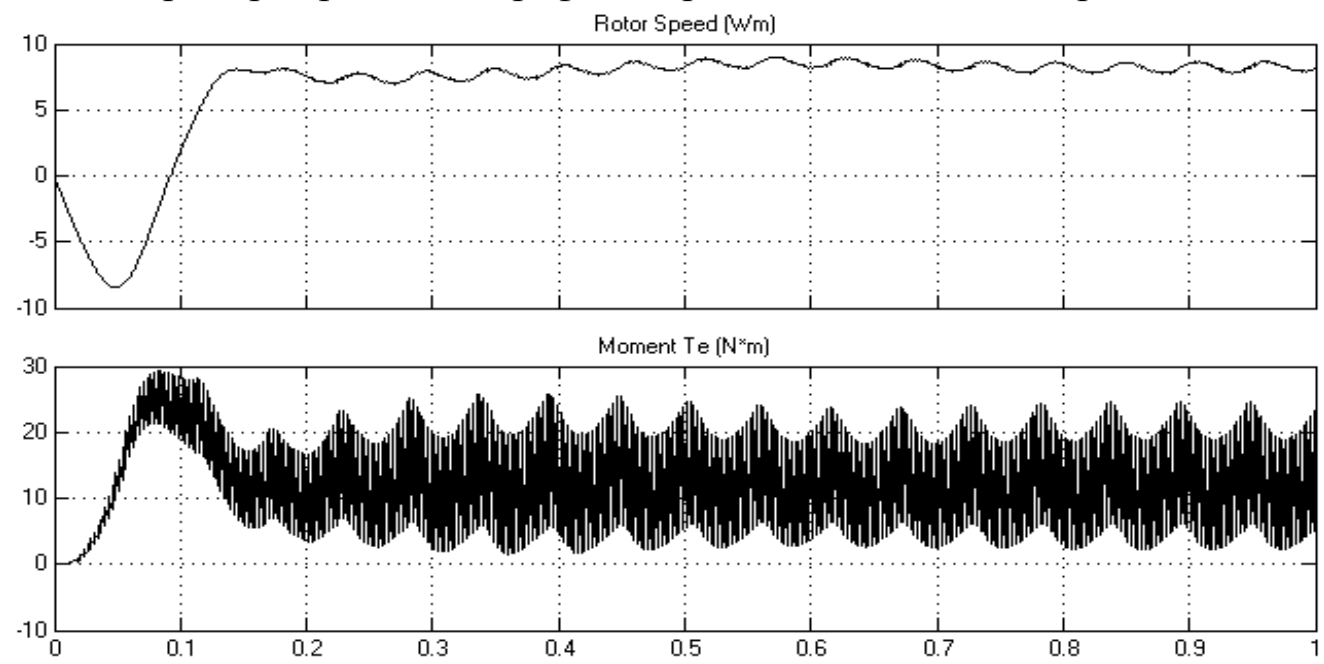

Рис. бСкорость ротораи электромагнитный момент двигателяпри $f=3, n=20$ 


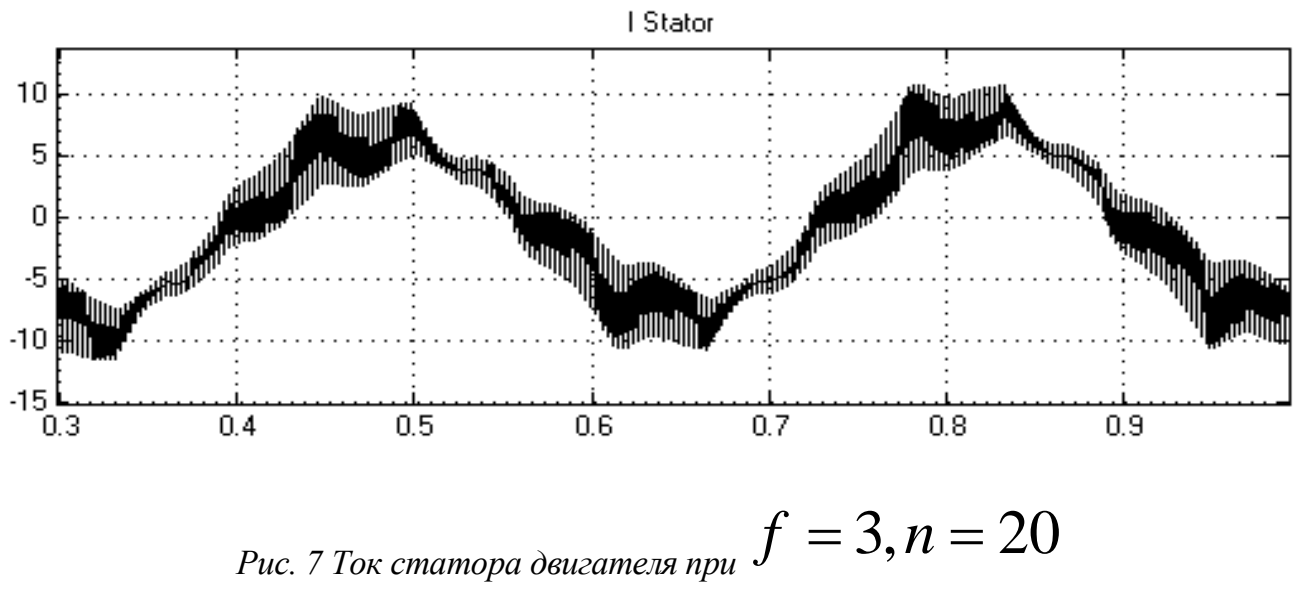

\section{Вывод}

Результаты моделирования регулирования частоты вращения показывают что колебание скорости, тока статора, электромагнитного момента двигателя уменщетсяпри больщем количестве формируемых векторов в каждом секторе чем при трационном. Таким образом, использование векторной ШИМ по предложенному алгоритму при времени существования промежуточных векторов существенно меньше относительно времени существования базовых векторов позволяет не только обеспечить все хорошие характеристики традиционного способа векторной ШИМ, но и устранить многие недостатки традиционного способа.

\section{Литература}

1. Борзов Д.Б., Титов В.С. Вопросы проектирования и динамической реконфигурации топологии систем логического управления в системах высокой готовности: монография / Юго-Зап. гос. ун-т. -Курск, 2015. -282 с.

2. Данильченко Н.В., Макеев С.Н. Масштабируемый мультипроцессор для цифровой обработки сигналов // Проблемы разработки перспективных микро- и наноэлектронных систем - 2012: сб. тр. /под общ. ред. акад. РАН А.Л.Стемпковского. - М.: ИППМ РАН, 2012. - С. 495-500. 